SPOR BİLIMLERİ ARAŞTIRMALARI DERGİSI

Journal of Sport Sciences Researches

http://dergipark.gov.tr/jssr

ISSN: 2548-0723

\title{
Hypothetical Approach to the Location of Genotypes (ACE \& ACTN3) Associated with Energy Systems for the Athletic Performance*
}

\author{
Mesut CERITT ${ }^{1, \dagger}$ \\ ${ }^{1}$ International Cyprus University, Nicosia/Cyprus, ORCID ID: 0000-0001-6910-4770
}

\begin{abstract}
This study has been done in order to reveal the theoretical approach about the energy corridor or pathway (the ability space in which every genotype is dominant) used by gene variables during the exercise or the training and the construction of the training loads related to the mentioned corridor.

In this study, under the umbrella of the studies having been done about genetic and sportive performance development so far, detailed literatüre review method has beeen utilized. Within the scope of this research, by analizing similar research found in literatüre, they have been interpreted and evaluated as goal-oriented.

The "model of the location of genotypes associated with the energy systems" will be a beneficial tool for the athletes and trainers for creating, establishing, constructing or organizing a training program or individiual oriented training programs.

Conclusion; genetic heritage, which triggers sport success, is blended with environmental factors, life style, application of the trainings, coverage and intensity of the loads, development of neuromotor, balanced nourishment and cultural differences and it reveals changes or differentiations which are peculiar to the individual. When all of these variables are considered, the performance development and sport success may be aligned to more Original Article foreseeable trajectories.

Original Article
Received: 06.05 .2018
Accepted: 19.06 .2018
Online Published: 30.06 .2018

DOI: $10.25307 /$ jssr.421427
\end{abstract}

\section{INTRODUCTION}

In today's world where the relationship between the genetics and the sport gain momentum, multi-disciplinary studies meet us with a new finding every passing day. When it comes to especially the relationship between sport performance and the gene, it is of great significance to reflect the obtained findings in a careful and thorough way to the sport world. In recent years there has been a great progress in molecular biology techniques, which has enhanced the influence of the research both on genetics and on human performance.

Advances in biotechnology have allowed investigating various genes and genetic markers that could possibly be associated with various fitness phenotypes (Gibson, 2009), such as muscle strength/size (Hubal et al., 2011) or aerobic capacity (Goḷeva, 2015; Pitsiladis et al., 2011). The concept that genetic traits are strongly associated with human physical performance has been widely accepted in the past decade (Yamak et al., 2015). Over the past decades, a large number of studies revealed various associations between specific genes and

\footnotetext{
* This study was presented as Oral Presentation in "International Balkan Sport Science Conference 2018" conference held in Tetowa/MACEDONIA, between 18-21 May, 2018.

† Sorumlu Yazar: Mesut CERIT, E-mail: mesutcerit@yahoo.com.
} 
their polymorphisms and physical ability in various sport fields and across different ethnicities and genders. There is increasing evidence for strong genetic influences on athletic performance and for an evolutionary "trade-off" between performance traits for speed and endurance activities (Yang et al., 2003). In this regard; there are specific regions of DNA that can vary amoung individuals. Such variations (i.e., polymorphisms) may, in part, explain why some individuals give differentiated responses to certain stimuli, including the responses to sports training. So, in a particular sport, the presence of specific polymorphisms may contribute to higher levels of performance (Guilherme et al., 2014).

Each gene contains a particular set of instructions, usually coding for a particular protein or particular function. Genes are effective in the creation and proper functining of the basic structures of the body such as heart, lungs, muscle, skeleton and blood. It's appearent that genetic factors are effective for these complex structures. Moreover, in creating extraordinary effects, the genetic factors influence the physiological structure at certain degrees (Guth \& Roth, 2013).

For instance, blood flow velocity which feeds our cells by circulating our body like a cobweb and the functioning of our strong and enduring muscles which help us move are also genetically coded as well. Researchers are now concentrating on looking for the exact genetic profiles that contribute to sport performance and determining the underlying mechanisms involved in specific fields of elite athletic performance. One of the main aims of such studies is to help clinicians and coaches to recognize and guide individuals with genetic potentiality to be evolved elite athletes (Ma et al., 2013).

This study has been done in order to reveal the theoretical approach about the energy corridor (the ability space in which every genotype is dominant) used by gene variables during the exercise or the training and the construction of the training loads related to the mentioned corridor.

In this study, we specifically consider two genes, which have been extensively studied for the association with athletic ability, namely, the angiotensin I-converting enzyme (ACE) and $\alpha$-actinin-3 (ACTN3). ACE and ACTN3 genes are among the most studied genes in the field of exercise genomics. They are thought to have influence on traits such as muscle strength, endurance and trainability. I-allele of the ACE gene, and X-allele for the ACTN3 gene has been generally associated with improved endurance, while D-allele and R-allele for the ACE and ACTN3 genes, respectively, has been associated with sprint and power related phenotypes (Goḷeva, 2015).

ACTN3 577R allele and ACE D allele dominant model account for $0.92 \%$ and $1.48 \%$ of sprint time variance, respectively. In spite of sprint performance relying on many gene variants and environment, the \% sprint time variance explained by ACE and ACTN3 is substantial at the elite level and might constitute the difference between a world record and only making the final (Papadimitriou et al., 2016). In this respect, the present study summarizes the associations of sport performance with ACE I/D and ACTN3 R577X polymorphisms. The results consistently provides more sound evidence for associations between ACE II genotype and endurance events, and between ACTN3 R allele and power events. Our findings provides well-founded evident to support that human physical 
performance might be influenced by genetic profiles (Ma et al., 2013). Meta-analysis, which analyzes the relationships between the ACE I/D, the ACTN3 R577X genotypes and athletic performance, conducted by Ma et al. (2013), has revealed significant associations of the ACE II genotype with enhanced endurance performance and the ACTN3 R allele with better power performance (Magi et al., 2016).

\section{METHOD}

In this study, under the umbrella of the studies having been done about genetic and sportive performance development so far, detailed literatüre review method has beeen utilized. Within the scope of this research, by analizing similar research found in literatüre, they have been interpreted and evaluated as goal-oriented.

One of the details which needs to be contemplated in training planning is that weekly training loads (intensity and volume) can accurately be determined by taking into consideration the dominant energy systems which are suitable for the sportsman's genetic structure and the sport's feature.

In figure 1, in which $\mathrm{ACE}$ and ACTN3 variables take place, it is depicted that dominant energy systems used during the training, exercise intensity and duration and both genotype features have different parameters rather the training fields in which they are dominant are shown.

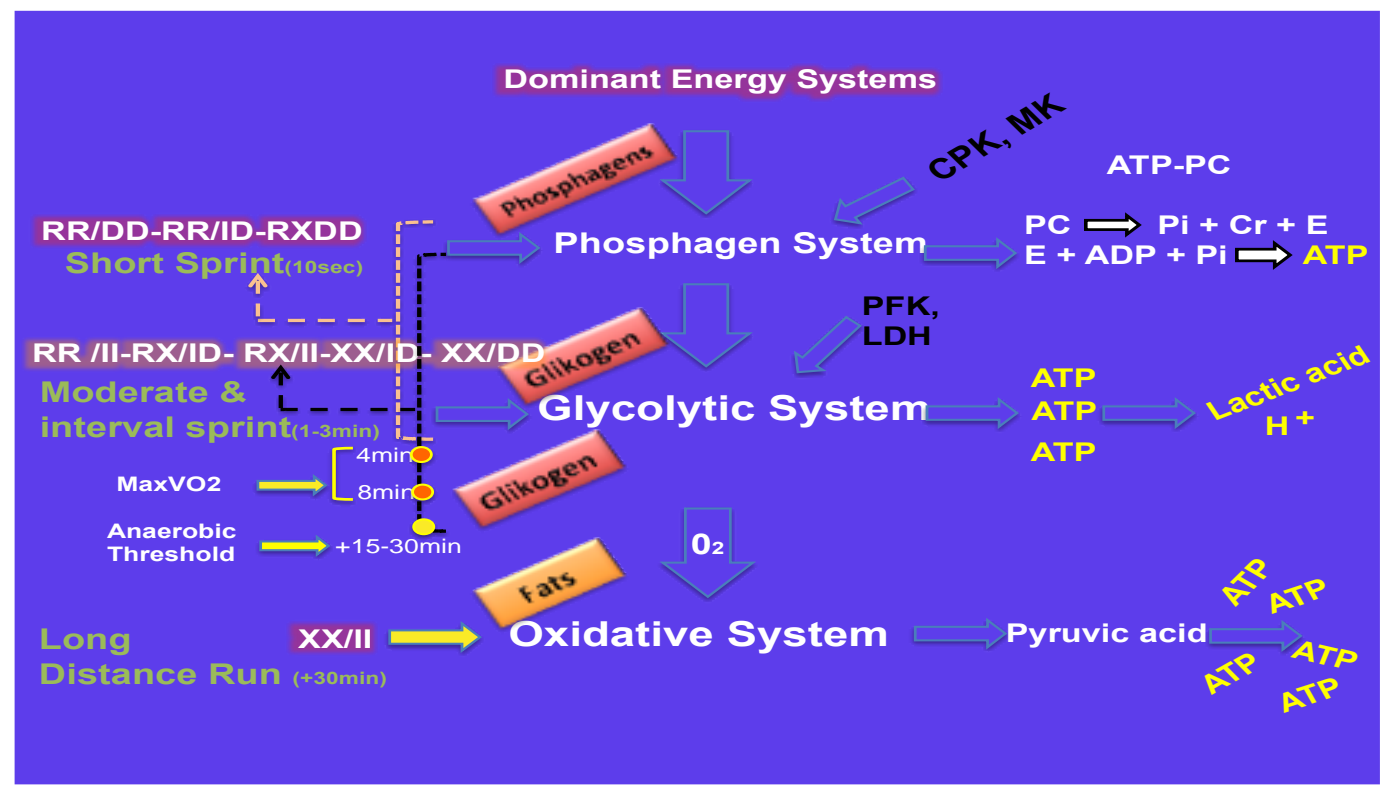

Figure 1. Hypothetical approach to the location of genotypes (ACE \& ACTN3) associated with energy systems for the athletic performance.

When we take into consideration using dominant energy system, individual genotype differences and location of genotypes (ACE \& ACTN3) associated with the type of muscle fibers and training zone for a microcyle in figure 1, 2 and 3 respectively, we can confirm the intensity and the duration of training loads by determining what kind of a training requirement that genotypes need. 
Cerit, M. (2018). Hypothetical Approach to the Location of Genotypes (ACE \& ACTN3) Associated with Energy Systems for the Athletic Performance. Journal of Sport Sciences Researches, 3(1), 97-105.

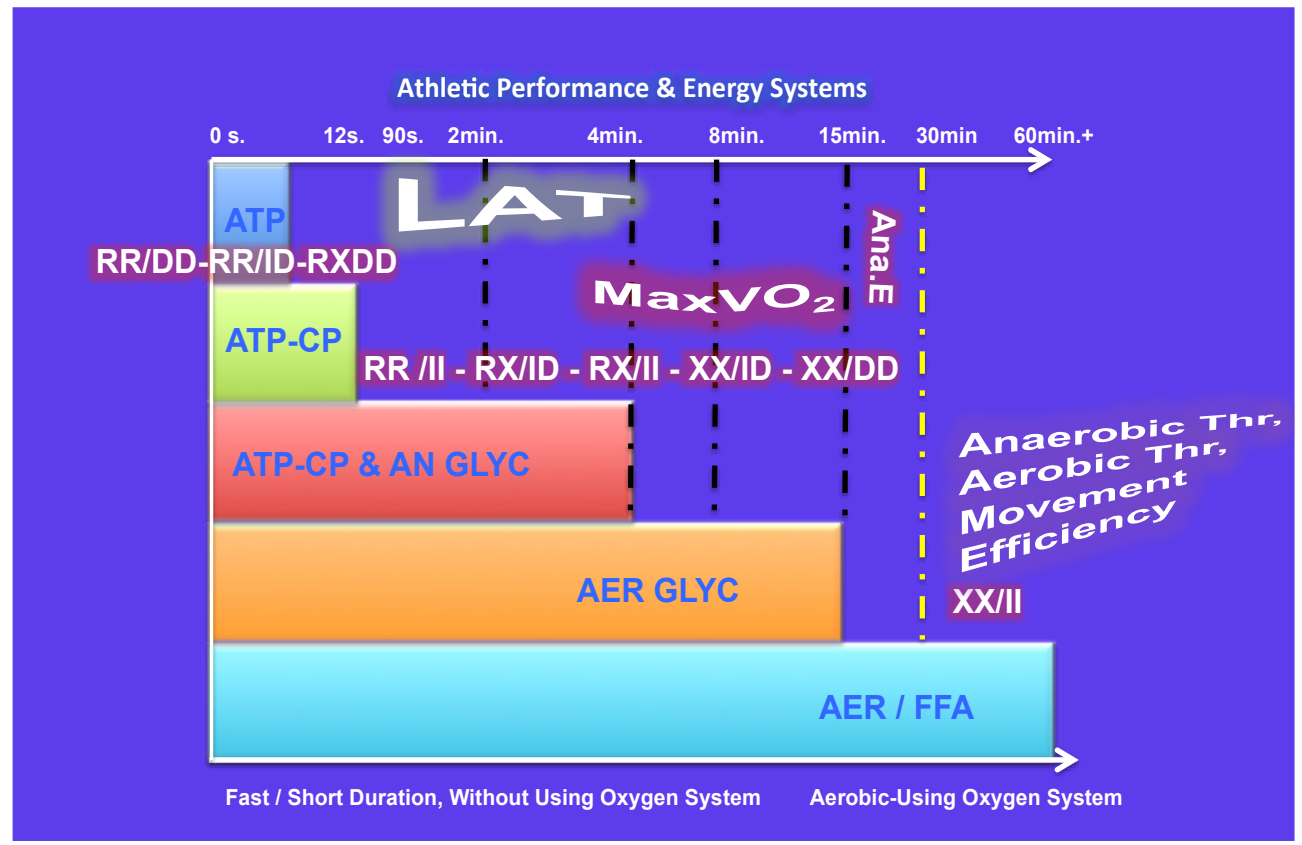

Figure 2. Hypothetical approach to the location of genotypes (ACE \& ACTN3) associated with training zone and energy systems for the athletic performance.

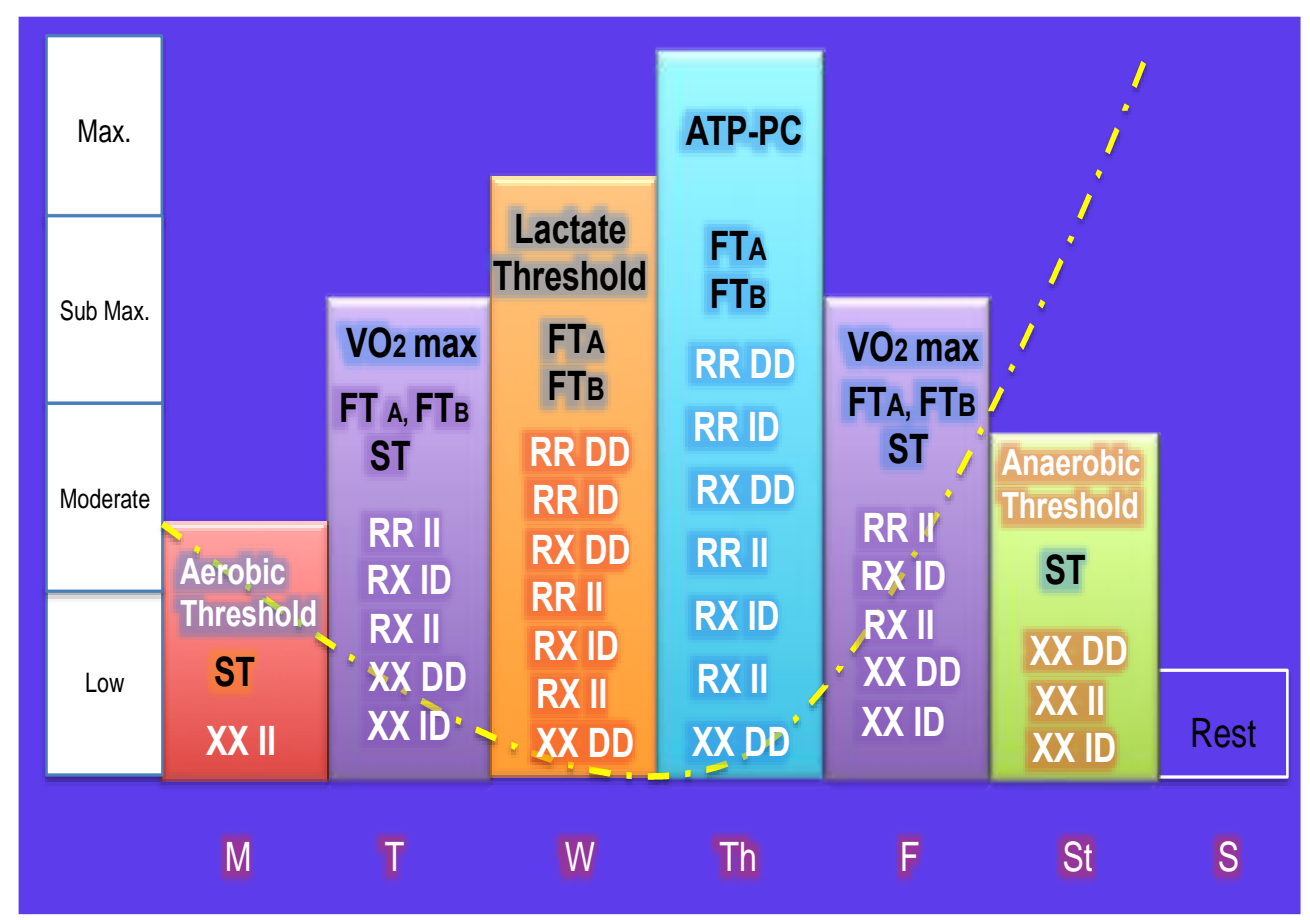

Figure 3. Hypothetical approach to the location of genotypes (ACE \& ACTN3) associated with the type of muscle fibers and training zone for a microcyle.

\section{RESULT}

Relying upon the aforementioned explanations and taking into consideration the findings which have been stated in previous studies, we can infer that individual differences in genetic terms is to considered while arranging and portraying a training program for the athletes and 
sportsmen. In taking into consideration that the one who has the right preparation at the right time is on the top, attaching importance to the genotypes features of sportsman will help us in both paving the way for ourselves to achieve the goals and using the time effectively. Thus, it's possible to eliminate or at least hinder the probable disappointments, which are likely to occur later. In this regard, the fitness programs should be individualized for participants. Not only do individual goals vary, but individuals also respond and adapt to exercise differently and one of the major determinants of the individual's response is genetics (Plowman \& Smith, 2011).

The "model of the location of genotypes included in the figures (1,2 \& 3) associated with the energy systems" will be a beneficial tool for the athletes and trainers for creating, establishing, constructing or organizing a training program or individiual oriented training programs.

\section{DISCUSSION}

ACE and ACTN3 genotype association differences may be related to subtle differences in the physiological performance demands of each event. In the $100 \mathrm{~m}$ race the athlete is required to accelerate for most of the race before reaching their absolute maximum velocity. In longer sprinting events (200 $\mathrm{m}$ and $400 \mathrm{~m}$ ) the acceleration phase is relatively shorter, and rather, it is the ability to maintain the maximum velocity for a longer time period that is the critical factor for winning the events. Acceleration relies on reaction time, centre of gravity of the body relative to the blocks, frequency of step and step length, while maintaining absolute maximal velocity requires powerful cyclic muscle contractions and efficient utilization of the energy systems (mostly lactic and 'alactic' anaerobic systems) that are triggered at different phases of the race. Given the genotype performance associations at longer ( $200 \mathrm{~m}$ and $400 \mathrm{~m}$ ) distances, this suggests their influence may lie greater effect on muscle's metabolic potential (switch from $\mathrm{P} / \mathrm{Cr}$ to lactic anaerobic systems) with repeated powerful contractions (Papadimitriou et al., 2016).

Between the 2nd and 4th minutes in short duration aerobic endurance (2-8 minutes), while the tolerance to lactic acid is more important, in determining the performance between the 4th and 8th minutes, the $\mathrm{VO} 2 \max$ becomes dominant. High performance in short duration aerobic performance (2-8min) demands high power output and increased tissue oxygenation. It requires higher $\mathrm{VO}_{2 \max }$ and strength endurance levels (Cerit, 2006; Cerit et al., 2006). There are similar variations in response to resistance training stimuli. Around $20-30 \%$ of both muscular fitness and $\mathrm{VO}_{2 \max }$ is thought to be genetically determined.

When it comes to medium duration aerobic endurance, (8-30 minutes), $\mathrm{VO}_{2 \max }$ is important between the 8th and 15th minutes, anaerobic endurance becomes dominant after 15th minute. I allele responses better to medium-duration (30 $\mathrm{min}$ ) aerobic endurance training (Cerit et al., 2006; Colakoglu et al., 2005).

As for the long duration aerobic endurance, (more than 30 minutes) performance factors such as anaerobic threshold, the amount of oxygen used in anaerobic threshold, aerobic threshold 
and movement efficiency become important (Cerit et al. 2006; Kraemer et al. 2012). Endurance performance depends mainly on the integration of cardiorespiratory fitness, metabolic processes in skeletal muscles and neurological factors, but biomechanical, psychological, physical, nutritional and other parameters are important as well (Kraemer et al., 2012; Magi et al., 2016). This genotype may therefore be advantageous in response to high-volume and low-intensity strength training (Colakoglu et al., 2005).

Variations in trainability have been observed in both the general population (Bouchard, 2012) and athletes performing at top level (Ahmetov et al., 2012). The ability to perform aerobic or anaerobic exercise varies widely among individuals, partially depending on their muscle fiber composition. Type I fibers (slow twitch oxidative fibers) have high resistance to fatigue (and are thus suited for endurance performance), type II a fibers (fast twitch oxidative glycolytic fibers) are better suited for medium-term anaerobic exercise, and type II b fibers (fast twitch glycolytic fibers) are adapted for short bursts of high speed and power (Ahmetov et al., 2012; Farrell et al., 2012; Kraemer et al., 2012). Endurance-oriented athletes are reported to have a remarkably high proportion of type I fibers in their trained muscle groups whereas muscles of sprinters and weight lifters predominantly consist of $\mathrm{IIa} / \mathrm{IIb}$ fibers (Ahmetov et al., 2012).

The characteristics of muscle fibers appear to be determined early in life, perhaps within the first few years. Studies with identical twins have shown that muscle fiber type, for the most part, is genetically determined, changing little from childhood to middle age. The genes we inherit from our parents likely determine which a motor neurons innervate our individual muscle fibers (Costill et al., 2012). As a general rule, the fast fibers are important for shortduration, high-intensity work bouts, whereas the slow fibers are better suited for submaximal, prolonged activities. The percentage of each of these major types in a given muscle appears to be genetically determined. Most muscles in the body contain a combination of fiber types, which is influenced by genetics (Plowman \& Smith, 2011; Kraemer et al., 2012) hormonal profile, training, and function of the muscle. Elite endurance athletes typically demonstrate a predominance of type I muscle fibers (e.g., 70-85\%), whereas elite sprinters typically demonstrate a predominance of type II muscle fibers (65-70\%), (Kraemer et al., 2012).

ACE and ACTN3 genotypes associated with the energy system for 5 different training zones (different training volume and intensity) in one-week microcyle. Dominant genotype RR/DD individual or athletes (sprinters) should arrange the weekly training plan by using at least one or two ATP-PC and lactate threshold training and one aerobic threshold for the regeneration and enhance aerobic endurance. These genotypes, since they have dominant fast twitch fiber (Type II b), their capacity to perform for the success should be trained anaerobic energy systems associated with the training zone (ATP-PC \& Lactate Threshold), otherwise genetics limitation may constitute an obstacle for the success. The important thing is what is your fuel is during the exercise (what the body needs for) and whats your genetic background is to burn from the different kinds of fuels that you dominantly have.

By determining the approximate rates of the fuels (phospogens, carbonhydrate, fat and protein) that your bodies will need before, during and after the training-regeneration phase, you should fix the training loads. You can enable effective sport performance development 
taking into consideration the features of the sport itself and sportsman's genetic features-by planning more aerobic loads in number to the individuals having higher levels of slow twitch fiber and planning more anaerobic loads to the individuals having higher levels of fast twitch fiber.

Determination of the branchs of the athletes according to the specification in their genes (with reference to figure 1 and 2); The determinants of human athletic performance have long been a challenging field of study in sport sciences. Sports performance is an enormously complex multifactorial phenomenon, and is usually determined not only by numerous intrinsic (e.g., genetics, motor behavior, physiological and psychological profile) and extrinsic factors (e.g., training, nutrition, development opportunities and overall health conditions) but also by various interactions between them. Notwithstanding it is impossible to set a unique formula to make anyone becoming a successful athlete, it is widely accepted that any individual who is highly committed and dedicated to training is able to improve athletic performance. Likewise, to be a top-level athlete, several years of dedication to an organized and rigid training system is indeed a prerequisite, although not a guarantee for success. However, a few athletes seem to be exceptionally gifted and demonstrate extraordinarily high performance levels even before taking part in training programs; some athletes demonstrate better responses to training than others, or may be able to consistently sustain high levels of performance over their competitive career (Guilherme et al., 2014).

High anabolic genotypes (RR/DD); preliminary or from the beginning of 20 seconds, have anabolic muscular (high) and anabolic cardiovascular system (high); short distance run (50m, $100 \mathrm{~m}, 60 ; 100 ; 110 \mathrm{~m}$ hurdle, 200m sprinters), shot put, discus throw, hummer throw, jawelin throw, weight lifting, power lifting, body building and short distance swimming are the examples of the events for high anabolic genotypes.

For moderate anabolic genotypes which are dominant until 90 seconds, anabolic muscular system (high), anabolic cardiovascular system (moderate) (RR/DD); anabolic muscular system (moderate), anabolic cardiovascular system (high) (RX/DD); 400m run, 400m hurdle run, long jump, speed scating, Judo, fitness, boks, wrestling, rugby, taekwondo, 400m swimming etc. are the examples of the events for moderate anabolic genotypes.

For power and endurance genotypes which are dominant or effective until 15 minutes, muscular strength, cardiovascular endurance, (RR/II; RX/ID; RX/II), muscular endurance, cardiovascular strength, (XX/ID; XX/DD); 5000, $10.000 \mathrm{~m}$ distance run, american football, table tennis, cricket, tennis, football, water polo, volleyball, basketball, gymnastics, speed skating, ice hockey, rowing, kick boks etc. are the examples of the events for moderate anabolic genotypes.

High endurance genotype, genotypes which are effective in 60 minutes or more, muscular endurance, cardiovascular endurance (XX/II); cross-country ski, biathlon, climbing, field hokey, scuba diving, cycling, shooting, triathlon, marathon, long distance run (ultra trail) swimming and archery are the examples of the events for high endurance genotypes.

High performance in long duration aerobic performance (45-60 min) demands high power output and increased tissue oxygenation. It requires higher $\mathrm{VO}_{2 \max }$ and strength endurance 
levels (Kraemer et al., 2012; Rigat et al., 1992). The physiological characteristics of elite cross-country skiers are a result of both genetics and adaptation to rigorous multiyear, yearround training programmes. The aerobic capacity and upper body anaerobic power seem to be the most important physiological factors of success in winter endurance sports including cross-country skiers, biathletes, (Orysiak et al., 2013).

\section{CONCLUSION}

Genetic heritage, which triggers sport success, is blended with environmental factors, life style, application of the trainings, coverage and intensity of the loads, development of neuromotor, balanced nourishment and cultural differences and it reveals changes or differentiations which are peculiar to the individual. When all of these variables are considered, the performance development and sport success may be aligned to more foreseeable trajectories.

Sports performance is an enormously complex and multi-faceted phenomenon, and is determined by numerous factors such as genetics (proportion of muscle fiber type, aerobic and anaerobic power and capacity and adaptation to training), psychological and social factors, physical condition, knowledge and experience of the trainer, talent of the athlete and well planning as well as by the interaction among them. In this respect, learning or investigating individiual genotype features may represent a beneficial and utilizable tool for guiding the athlete towords coherent direction and the right events, creating individual oriented training programs and recognizing the individuals who have genetic potential for being elite athletes. Furthermore, this also promising opportunity in helping the trainers by giving them guiding information, whicih would enable the athletes to accomplish the optimum success and the ability to identify the physiological weaknesses of active athletes and designing the personal training programs accordingly. Consequently, you cannot change your genes, but you can activate/deactivate them or alter the way they are being used. The point is not the types but the way you trigger them is all that matters.

\section{REFERENCES}

Ahmetov, I. I., Vinogradova, O. L., \& Williams, A. G. (2012). Gene polymorphisms and fiber-type composition of human skeletal muscle. International Journal of Sport Nutrition and Exercise Metabolism, 22(4), 292-303. [Available online at: https://pdfs.semanticscholar.org].

Bouchard, C. (2012). Genomic predictors of trainability. Experimental Physiology, 97(3), $347-52$. DOI:10.1113/expphysiol.2011.058735.

Cerit, M. (2006). Relationship between ace genotypes and short duration aerobic performance development. PhD Thesis, Institute of Health Sciences, Sport Sciences Division, Ege University, Izmir, Turkey.

Cerit, M., Colakoglu, M., Erdogan, M., Berdeli, A., \& Cam, F. S. (2006). Relationship between ace genotype and short duration aerobic performance development. European Journal of Applied Physiology, 98(5), 461-465. DOI:10.1007/s00421-006-0286-6.

Colakoglu, M., Cam, F. S., Kayitken, B., Cetinoz, F., Colakoglu, S., Turkmen, M., \& Sayin, M. (2005). ACE genotype may have an effect on single vs multiple set preferences in strength training. European Journal of Applied Physiology, 95(1), 20-27. 
Cerit, M. (2018). Hypothetical Approach to the Location of Genotypes (ACE \& ACTN3) Associated with Energy Systems for the Athletic Performance. Journal of Sport Sciences Researches, 3(1), 97-105.

Kenney, L., Wilmore, J., \& Costill, D. (2012). Physiology of sport and exercise (5th ed). USA: Human Kinetics. pp. 41-42.

Farrell, P.A., Joyner, M.J., \& Caiozzo, V.J. (2012). ACSM's Advanced exercise physiology (2nd ed). Hong Kong: Wolters Kluwer. pp. 39-40

Gibson, W. T. (2009). Key concepts in human genetics: understanding the complex phenotype. Medicine and Sport Science Journal, 54, 1-10. DOI:10.1159/000235693.

Goḷeva, F. S. (2015). Genotype frequency distribution of ACE I/D and ACTN3 R577X polymorphisms in the Norwegian population. Do ACE I/D and ACTN3 R577X polymorphisms influence self-reported physical activity levels. Master's Thesis in Environmental Health and Science. [Available online at: https://brage.bibsys.no/xmlui/handle/1].

Guilherme, F., Tritto, C., North, N., Lancha, H., \& Artioli, G. (2014). Genetics and sport performance: Current challenges and directions to the future. The Revista Brasileira de Educação Física e Esporte, 28(1), 177-93. [Available online at: http://www.scielo.br/scielo.php?script].

Guth, L.M., \& Roth, S.M. (2013). Genetic influence on athletic performance. Current Opinion in Pediatrics, 25(6), 653-658. DOI:10.1097/MOP.0b013e3283659087.

Hubal, M. J., Urso, M. L., \& Clarkson, P, M. (2011). Genetic aspects of muscular strength and size. In: Pescatello L., Roth S. (eds) Exercise Genomics. Humana Press. DOI: 10.1007/978-1-60761-355-8 7.

Kraemer, W.J., Fleck, S.J., \& Deschenes, M.R. (2012). Exercise physiology (1st ed). Baltimore: Lippincott Williams \& Wilkins. pp. 75-78.

Ma, F., Yang, Y., Li, X., Zhou, F., \& Gao, C. (2013). The association of sport performance with ACE and ACTN3 genetic polymorphisms, a systematic review and meta-analysis. PloS one, 8(1), 1-9. DOI: 10.1371/journal.pone.

Magi, A., Unt, E., Prans, E., Raus, L., Eha, J., Verekasits, A., Kingo, K., \& Köks, S. (2016). The association analysis between ACE and ACTN3 genes polymorphisms and endurance capacity in young cross- country skiers. Journal of Sports Science Medicine, 15(2), 287-294. [Available online at: https://www.ncbi.nlm.nih.gov/pubmed].

Orysiak, J., Zmijewski, P., Klusiewicz, A., Kaliszewski, P., Malczewska-Lenczowska, J., Gajewski, J., \& Pokrywka, A. (2013). The association between ace gene variation and aerobic capacity in winter endurance disciplines. Biology of Sport Journal, 30(4), 249-53. DOI: 10.5604/20831862.1077549.

Papadimitriou, I. D., Lucia, A., Pitsiladis, Y. P. Pushkarev, V. P., Dyallov, D. A., \& Orekhov, E. P. (2016). ACTN3 R577X and ACE I/D gene variants influence performance in elite sprinters: a multi-cohort study. BMC Genomics, 13 (17), 285. DOI: $10.1186 / \mathrm{s} 12864-016-2462-3$.

Pitsiladis, Y., Wang, G., \& Wolfarth, B. (2011). Genomics of aerobic capacity and endurance performance: clinical implications In: Pescatello, L.S. and Roth, S.M., eds. Exercise Genomics. Molecular and Translational Medicine Series. New York: Humana Press. pp. 179-230. ISBN 9781607613541.

Plowman, S.A., \& Smith, D.L. (2011). Exercise physiology for health, fitness and performance (3rd ed). Baltimore: Lippincott Williams \& Wilkins. pp.70-71.

Rigat, B., Hubert, C., Corvol, P., \& Soubrier, F. (1992). PCR detection of the insertion/deletion polymorphism of the human angiotensin converting enzyme gene (DCP1) (dipeptidyl carboxypeptidase 1). Nucleic Acids Res, 20(6), 1433. DOI: $10.1093 /$ nar/20.6.1433-a.

Yamak, B., Yüce, M., \& Bağc1, H., Imamoğlu, H. (2015). Association between sport performance and alpha-actinin-3 gene R577X polymorphism. International Journal of Human Genetics, 15(1), 13-19. DOI: 10.1080/09723757.2015.11886246.

Yang, N., Macarthur, D. G., Gulbin, J. P., Hahn, A. G., Beggs, A. H., Easteal, S., \& North, K. (2003). ACTN3 genotype is associated with human elite athletic performance. American Journal of Human Genetics, 73(3), 627-631. DOI:10.1086/377590. 